\title{
Comparison of intrusion errors and serial position curves on monaural and dichotic listening tasks: A developmental analysis
}

\author{
DOUGLAS FRIEDRICH* \\ Central Michigan University, Mount Pleasant, Michigan 48899
}

\begin{abstract}
Based on a repeated measurement study of 7-, 10-, 14-, and 17-year-olds with monaural and dichotic presentations of word lists varying in associative structure and presentation rate, intrusion and serial position dependent variables were analyzed. Intrusion analyses supported previous reports on word list recall; vis, 7- and 10-year-olds recalled fewer words from monaural and dichotic lists and had more intrusive errors than adolescents. Based on similar orders of report (strategies) for monaural and dichotic word presentations, serial position curves for the two types of presentation were compared. Within-age comparisons were strikingly similar. Between-age comparisons of monaural and dichotic presentation serial position curves support the notion that there are ontogenetic limitations in memory structure and control processes.
\end{abstract}

In two reports of a repeated measurement study of children and adolescents under monaural and dichotic word list presentation tasks, Friedrich (in press, a, b) provided a developmental framework to memory structure and control process models. Specifically, Friedrich reported findings which indicated ontogenetic limitations in both structural (short-term memory) and control process (strategies) components. In this report, two analyses with 7-, 10-, 14-, and 17-year-old males and females are summarized: intrusion errors and serial position curve comparisons of $S$ performance with monaural and dichotic word list presentation under conditions of associative structure and presentation rate.

\section{METHOD}

\section{Subjects}

The Ss were 80 students from a rural school district in Michigan, with 10 males and 10 females in each of the following groups: 7-, 10-, 14-, and 17-year-olds. Only right-handed Ss participated. Intelligence test results (Kuhlmann-Anderson) were used to restrict the sample to the normal IQ range. All Ss had been tested within 1 year of the study. No significant differences in IQ were found between or within age groups.

\section{Procedure}

The apparatus used to administer the stimuli consisted of a Sony two-channel tape recorder played into a pair of Sharpe headphones. Interstimulus intervals and dichotic presentation of stimuli were established by converting time intervals into tape speed intervals. Thirty-two eight-item lists were recorded under the following conditions, with four lists of associated and unassociated nouns per condition: (A) dichotic, 1-sec presentation rate; (B) dichotic, 3-sec presentation rate; (C) monaural, 1-sec presentation rate; and (D) monaural, 3-sec presentation rate. The words used to construct the associated word lists were 64 pairs of nouns. The first member of each pair was a Kent-Rosanoff stimulus word. The second member of each pair was the response to the first word given by the majority of

*Requests for reprints should be sent to the author, Department of Psychology, Central Michigan University, Mount Pleasant, Michigan 48859.
Ss in all age grades of the Minnesota norms (Palermo, 1965; Palermo \& Jenkins, 1964). ${ }^{1}$ To construct the associated lists, the 64 pairs of words were assigned at random, four pairs to a list. The ordering of the pairs within a list was also random. The word lists were recorded dichotically and monaurally at the rates of one pair $/ 1$ and $3 \mathrm{sec}$. The monaural lists were recorded such that they appeared in each channel equally often. Under the dichotic associative structure condition, the pairs were constructed so that each Kent-Rosanoff stimulus word (Channel 1) was paired with its associated response word (Channel 2). Under the monau ral associative structure condition, the pairs were constructed so that each Kent-Rosanoff stimulus word was followed by its associated response word. Pairs of unassociated lists were constructed by switching the associated response words used in Condition A with those in D and those used in B with those in C. Each S received 32 lists. The lists were presented in a partially counterbalanced order; half of the $S s$ in each age-sex group received $A$ first, the other half received D first.

The following scoring procedures from Bryden (1969) were used with Input Dichotic Pairs 1 (Channel 1), 2 (Channel 2), 3 (Channel 1), 4 (Channel 2), 5 (Channel 1), 6 (Channel 2), 7 (Channel 1), and 8 (Channel 2) as examples: (a) temporal order-reporting dichotic pairs as received, 1-2: 3-4: 5-6: 7-8; (b) ear order-reporting first those items presented to one ear and then those presented to the other, 1-2-3-4: 5-6-7-8; (c) attempted ear order-attempting to use ear order but failing to assign the items to the proper channel (transposition of a single pair of items produces ear order), 1-2-3-8: 5-6-7-4; (d) at tempted ear order with an "in place" repetition-attempting ear order but encoded items from one ear includes one item from each pair (transposition results in ear order with one error), 1-2-7-4: 5-6-7-8; and (e) random order-lack of any systematic response order over trials. When the $\mathrm{S}$ combined two strategies (e.g., temporal first, then ear), it was scored accordingly (e.g., temporal plus ear). Following the scoring method of Glanzer (1969) for serial lists, total number of correct responses for the four lists under each associative structure, presentation rate condition at each serial position was computed for each S. Orders of report for the monaural conditions were coded as follows: $\mathrm{B}=$ back (end), $\mathrm{BM}=$ back-middle, $\mathrm{BF}=$ back-front, $\mathrm{BFM}=$ back-front-middle, $\mathrm{BMF}=$ back-middle-front, FMB = front-middle-back, $\mathrm{MD}=$ middle-back, and random (miscellaneous) serial positions. To clarify the operation used to define strategies, B refers to recall of the last presented list words, $M$ refers to recall of words presented in the 
Table 1

Mean Intrusions for Each Age Group Under Dichotic and Monaural Word Conditions

\begin{tabular}{lrrrr}
\hline & & \multicolumn{3}{c}{ Conditions } \\
\cline { 3 - 5 } $\begin{array}{c}\text { Type of } \\
\text { Intrusion }\end{array}$ & Age & Dichotic & Monaural & $\begin{array}{c}\text { Dichotic }+ \\
\text { Monaural }\end{array}$ \\
\hline Associative & 7 & 1.45 & 2.15 & 3.60 \\
& 10 & 2.15 & 2.43 & 4.60 \\
& 14 & 1.15 & 1.20 & 2.35 \\
& 17 & 1.30 & 1.20 & 2.50 \\
Acoustic & 7 & 1.85 & 1.55 & 3.40 \\
& 10 & 1.20 & 1.30 & 2.50 \\
& 14 & 1.35 & 1.05 & 2.40 \\
Carry-Over & 17 & 1.50 & .90 & 2.40 \\
& 7 & 2.30 & 3.05 & 5.35 \\
& 10 & 2.60 & 2.50 & 5.10 \\
Miscellaneous & 14 & 1.20 & .90 & 2.10 \\
& 17 & .85 & .40 & 1.25 \\
& 7 & .60 & .60 & 1.20 \\
& 14 & .10 & .35 & .45 \\
Total & 17 & .10 & .00 & .10 \\
& 7 & 6.20 & .15 & .60 \\
$\Sigma$ & 10 & 6.05 & 6.35 & 13.55 \\
& 14 & 3.80 & 3.15 & 12.63 \\
& 17 & 4.10 & 2.65 & 6.90 \\
& & 20.15 & 19.73 & \\
\hline
\end{tabular}

middle of the list, and $F$ refers to recall of initial words presented in the list. For example, the B strategy represents immediate $^{2}$ recall of the last presented list words, the BM strategy represents immediate recall of the last presented words followed by middle list words, and the BMF strategy represents immediate recall of end list words followed by middle and then initial list words.

\section{RESULTS AND DISCUSSION}

The first analysis was done on intrusion errors. Mean intrusions (Kintsch, 1970)-acoustic, associative, list carry-over, and miscellaneous-for each age group under dichotic and monaural word list conditions are presented in Table 1. The following between-age significant differences were found (Scheffé, $\mathrm{p}<.05$ ): (a) total intrusion errors $-7,10>14,17$; (b) list carry-over errors $-7, \quad 10>14,17$; and (c) associative errors $-10>14,17$. No significant between-age differences were found for acoustic and miscellaneous errors. The above results hold for monaural, dichotic, and monaural plus dichotic list intrusions. No significant within-age differences were found comparing dichotic with monaural list performance. The significant between-age differences found for total intrusion errors reflect the dichotic and monaural word list recall performance of these groups (Friedrich, in press, a, b) $(7 \leqslant 10<14,17)$. Children recalled fewer words from dichotic and monaural word lists and made more intrusive errors than adolescents. In terms of specific intrusion errors, children indicated significantly more carry-over errors than adolescents. That is, recall of 7 and 10-year-olds contained significantly more words from previous lists than recall of adolescents. Ten-year-olds produced significantly more associated nonlist words than either younger or older Ss.

The carry-over intrusions illustrate the differential effect of irrelevant information interference on age groups, i.e., reflecting age-related change in control processes for information transfer from short-term to long-term memory. Children produced significantly more carry-over intrusions than adolescents, intrusions that were caused by prior presentation of these words. The associative intrusions findings suggest that 10-year-olds were affected more by extraexperimental learned associations than other age groups. Both carry-over and associative intrusions may be interpreted within the proactive interference model (Kintsch, 1970) or Atkinson's postulate of control processes associated with long-term memory. According to Atkinson and Wickens (1969) and Shiffrin and Atkinson (1969), long-term memory control processes are primarily involved with information storage and strategy selection. Further, the ability to retrieve stored information varies with time and interfering material. Basically, the long-term memory control processes aspect of Atkinson's model incorporates both decay (time) and interference (proactive, retroactive) theories of forgetting. Finally, the age-related intrusion findings
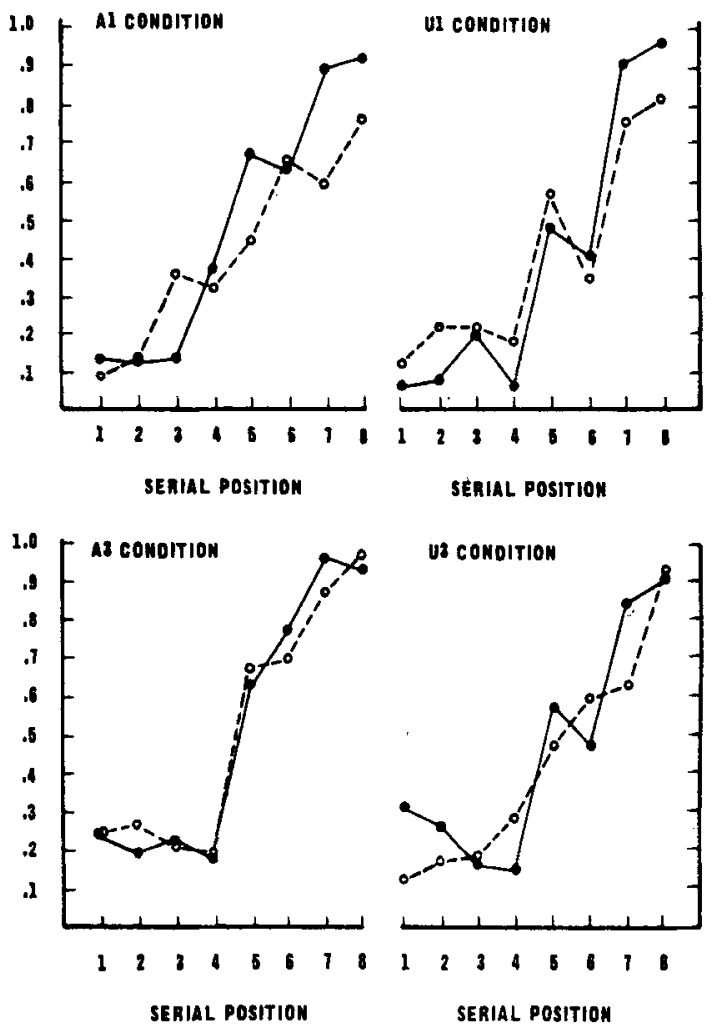

Fig. 1. Seven-year-olds' serial position curves for dichotic (open circle) and monaural (closed circle) presentation procedures. $\mathbf{A 1}$ = associated, $1-\sec$ presentation; $\mathbf{A} 3$ = associated, 3-sec presentation; U1 = unassociated, $1-\sec$ presentation; U3 = unassociated, 3 -sec presentation rate conditions. 
argue against the use of static memory models (Friedrich, 1972) and emphasize the need for descriptive-normative research. The present findings and those of Friedrich (in press, a, b) indicate ontogenetic limitations for memory structure capacity and control processes.

The second comparative analysis was done with serial position curves. Dichotic and monaural presentation serial position curves are presented in Figs. 1-4. It is difficult to compare serial position curves for dichotic and monaural lists since, in the dichotic list, pairs of words were presented simultaneously. Which is the first serial position? One reasonable solution is to compare serial position curves for dichotic and monaural lists in terms of output order in recall, as the back-to-front strategy is dominant in both presentation procedures. That is, in the Friedrich (in press, a, b) reports, the dominant strategy used by all age groups in both dichotic and monaural task presentation was recalling end list words first, followed by middle and front position words. One exception to this dichotic-monaural match-up was the moderate to high employment of ear orders of report under the unassociated, $1 \cdot \mathrm{sec}$
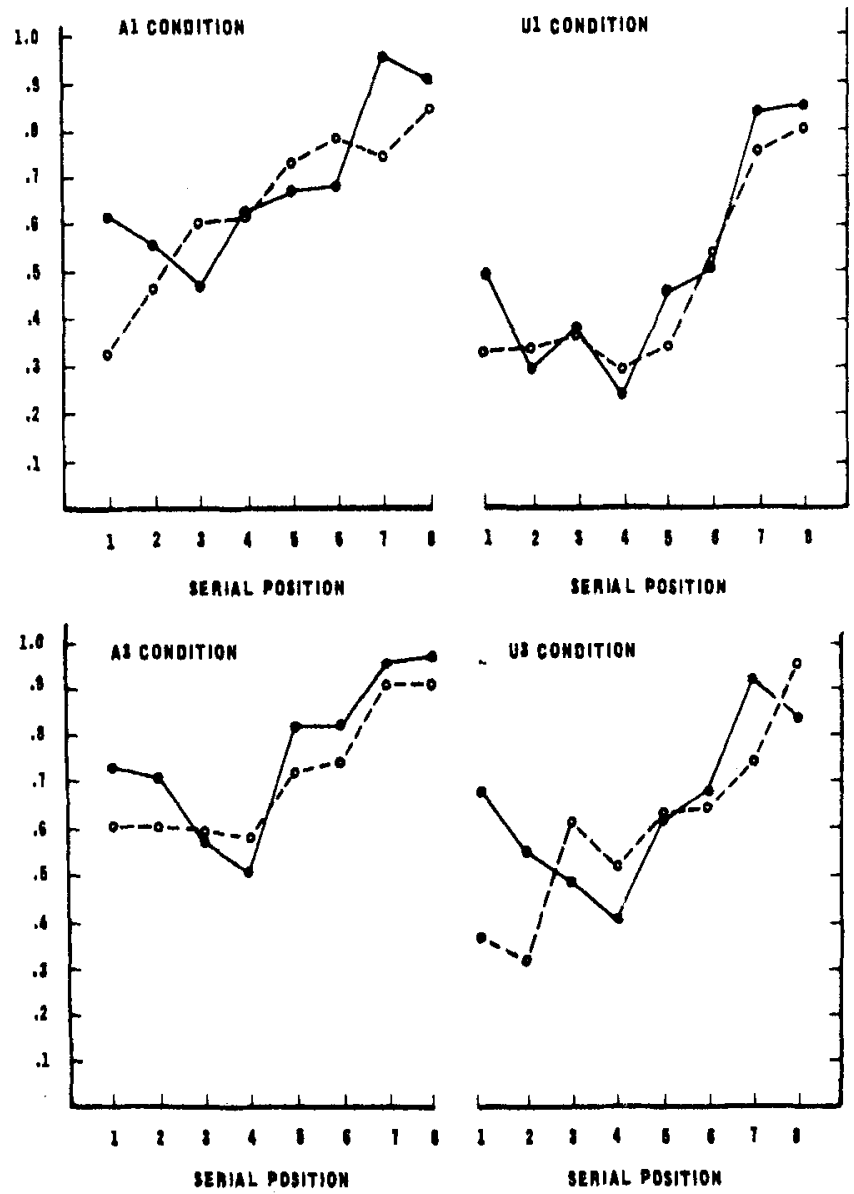

Fig. 2. Ten-year-olds' serial position curves for dichotic (open circle) and monaural (closed circle) presentation procedures. A1 $=$ associated, 1-sec presentation; $\mathbf{A 3}=$ associated, 3-sec presentation; $\mathrm{U1}=$ unassociated, $1-\sec$ presentation; and $\mathrm{U} 3$ = unassociated, 3-sec presentation rate conditions.
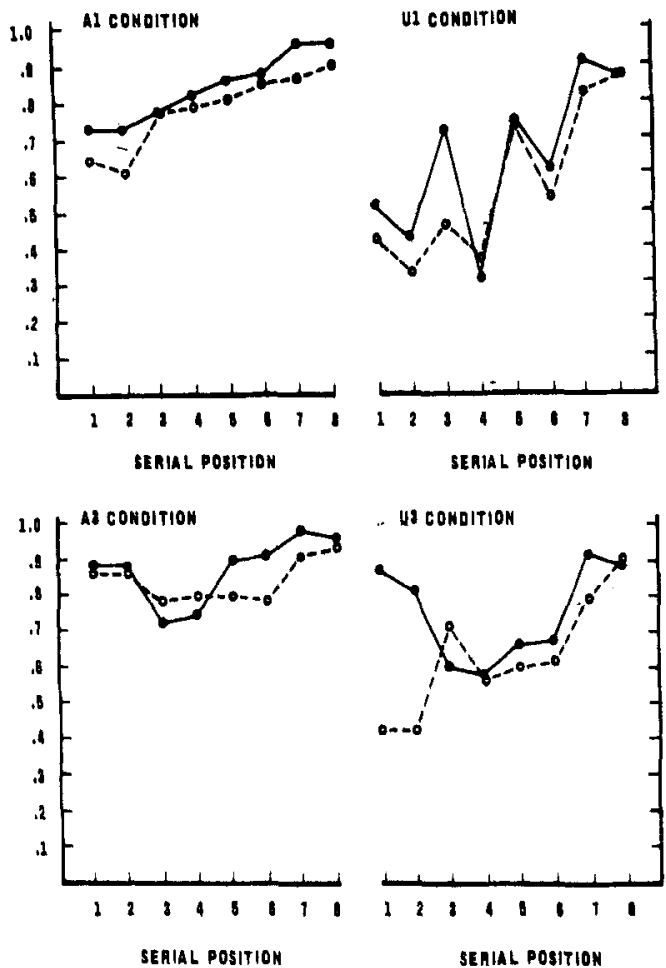

Fig. 3. Fourteen-year-olds' serial position curves for dichotic (open circle) and monaural (closed circle) presentation procedures. $\mathbf{A l}=$ associated, $1-\sec$ presentation; $\mathbf{A 3}=$ associated, 3-sec presentation; $\mathrm{U1}=$ unassociated, 1 -sec presentation; and U3 = unassociated, 3-sec presentation rate conditions.

presentation rate dichotic condition (recalling words from Channel 1 and then Channel 2). The curve matching in Figs. 1-4 incorporates the ear strategies under the unassociated, 1-sec presentation rate condition and is discussed below. For all age groups, serial position curves for associated, 1- and 3-sec presentation rate and unassociated, $3-\mathrm{sec}$ presentation rate dichotic conditions were plotted in the following manner. Serial Position (SP) 1 equals output from Secondary Channel (SC) 4 , that is, SP1 refers to the first word in the second half-span or channel recalled. Employing the temporal backward strategy, the $\mathrm{S}$ recalled the first word in the second half-span last. Likewise, the first word in the first half-span (primary channel, PC) was recalled second to last (SP2). This plotting procedure simply indicates that words recalled initially in free recall are plotted at the end positions of the abscissa and words recalled last are plotted at the beginning positions of the abscissa. This procedure for dichotic word lists reflects the basis for monaural word list serial position curves, i.e., end serial positions represent output from short-term memory (words immediately recalled) and beginning and middle serial positions represent output from long-term memory (Glanzer, 1969). The dichotic word list serial position curves for the associated, 1- and 3-sec presentation rate and unassociated, $3-\mathrm{sec}$ presentation rate conditions reflect the following response output: SP1 $=\mathrm{SC} 4$, $\mathrm{SP} 2=\mathrm{PC} 4, \quad \mathrm{SP} 3=\mathrm{SC} 3, \quad \mathrm{SP} 4=\mathrm{PC} 3, \quad \mathrm{SP} 5=\mathrm{SC} 2$, $\mathrm{SP} 6=\mathrm{PC} 2, \quad \mathrm{SP} 7=\mathrm{SC} 1$, and $\mathrm{SP} 8=\mathrm{PC} 1$. Thus, $\mathrm{SP} 1$ 

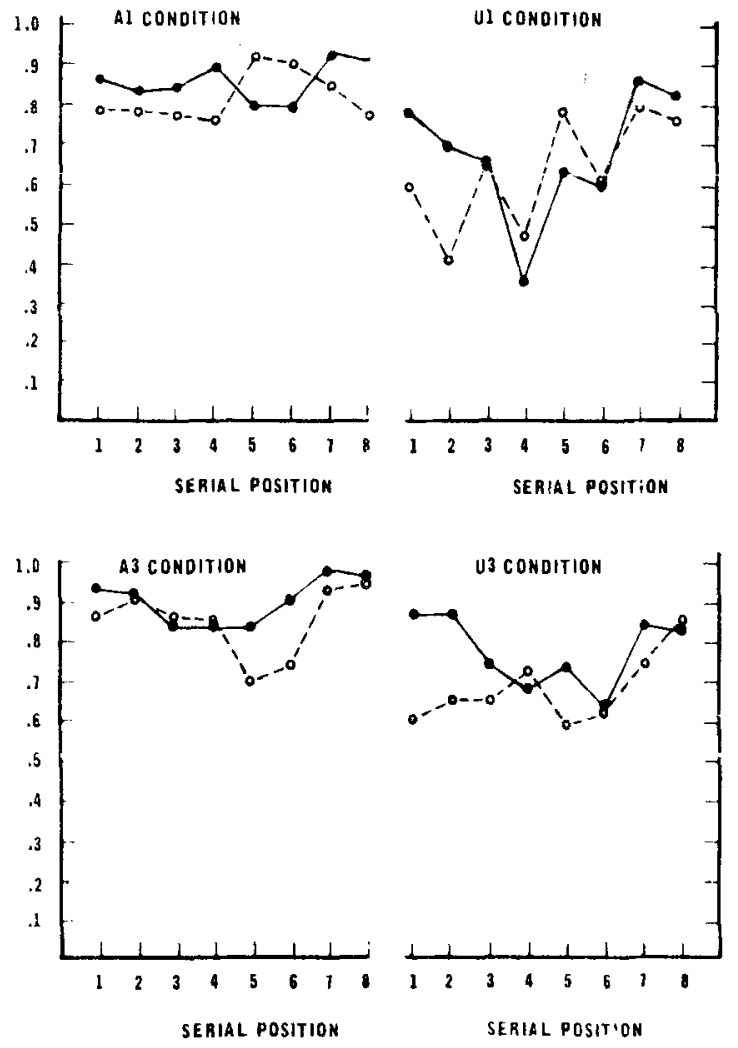

Fig. 4. Seventeen-year-olds' serial position curves for dichotic (open circle) and monaural (closed circle) presentation procedures. A1 = associated, $1-\sec$ presentation; $\mathbf{A} 3$ = associated, 3-sec presentation; U1 = unassociated, 1-sec presentation; and U3 = unassociated, 3-sec presentation rate conditions.

represents recall accuracy of the last presented word in the SC, SP2 represents recall accuracy of the last presented word in the PC, etc. (reflecting the temporal backward order of report). For all age groups, serial position curves for the unassociated, 1-sec presentation rate condition (ear strategies) reflect the following plotting procedures: $\mathrm{SP} 1=\mathrm{SC} 1, \mathrm{SP} 2=\mathrm{SC} 2, \mathrm{SP} 3=\mathrm{SC}$, $\mathrm{SP} 4=\mathrm{SC} 4, \quad \mathrm{SP} 5=\mathrm{PC} 1, \quad \mathrm{SP} 6=\mathrm{PC} 2, \quad \mathrm{SP} 7=\mathrm{PC} 3$, and SP8 $=$ PC4. Here, the first four positions represent recall accuracy for the four words from Channel 2 and the last four positions represent recall accuracy for the four words from Channel 1. Again, with the ear order of report for the unassociated, 1-sec presentation rate condition, the serial position curve reflects the plotting of words according to response output. Thus, the ear order requires a different plotting procedure for the dichotic, unassociated, $1-\mathrm{sec}$ presentation rate condition than the remaining conditions. It is noted, however, that both plotting procedures used reflect similar order of list word output; vis, end serial positions reflect words first recalled, middle positions reflect the next set of words recalled, and beginning serial positions reflect the last set of words recalled.

The dichotic and monaural curves are strikingly similar, with several apparent discrepancies. The only consistent discrepancy was in the unassociated, 3-sec presentation rate condition. For 14-year-olds the unassociated, 3-sec presentation rate discrepancy between dichotic and monaural curves at Position 3 reflects the relatively large difference in recall under dichotic-monaural conditions (.62) in favor of monaural presentation. Under the unassociated, $3-\mathrm{sec}$ presentation rate condition, the discrepancy between monaural and dichotic curves at Serial Positions 1 and 2 increases with increasing age. This finding reflects the increasing difference (monaural $>$ dichotic) in total amount recalled between presentation procedures. The discrepancy in the unassociated, 3-sec presentation rate condition curves at the beginning serial positions (.38, $.44, .95$, and .77 for ages $7,10,14$, and 17 , respectively) indicates that relatively slow dichotic presentation of words is a more difficult task than slow monaural presentation of words. More specifically, recall of initial list words under the unassociated, $3-\mathrm{sec}$ presentation rate condition was less accurate with dichotic presentation than with monaural presentation. The order of report summaries for dichotic and monaural presentation (Friedrich, in press, a, b) indicates that 10-, 14-, and 17-year-olds employed the front-to-back strategy with moderate to high frequency under the monaural unassociated, $3-\mathrm{sec}$ presentation rate condition but did not employ the strategy under the same dichotic condition. These strategy findings account for the increasing discrepancy between dichotic and monaural curves at Serial Positions 1 and 2 for the unassociated, 3 -sec presentation rate condition.

The overall consistency of dichotic and monaural curves under the four experimental conditions supports the magnitude of main effect coefficients for list structure variables of Friedrich (in press, $a, b$ ) for dichotic and monaural presentations. Similar magnitude coefficients were found in both presentation conditions for associative structure $\left(\mathrm{r}_{\mathrm{m}}=.80\right.$ dichotic, .80 monaural) and presentation rate $\left(\mathrm{r}_{\mathrm{m}}=.45\right.$ dichotic, .55 monaural). Within-age between-conditions relationships for words recalled under both presentation procedures are presented in Table 2. As illustrated in the table, with dichotic presentation a combination of structure variables (associative structure and 3-sec presentation rate) resulted in significantly greater recall for $7-, 10-$

Table 2

Between-Condition Relationships for Words Recalled Under Dichotic and Monaural Conditions at Each Age

\begin{tabular}{rll} 
& \multicolumn{2}{c}{ Presentation Procedures } \\
\cline { 2 - 3 } Age & \multicolumn{1}{c}{ Dichotic } & \multicolumn{1}{c}{ Monaural } \\
\hline 7 & A3 $>$ A1, U3, U1 & A3, A1 $>$ U3 > U1 \\
10 & A3 $>$ A1, U3, U1; & A3 $>$ A 1 $>$ U3 > U1 \\
& A1, U3 > U1 & \\
14 & A3 > A1 > U3 > U1 & A3, A1 > U3 > U1 \\
17 & A3, A1 $>$ U3, U1 & A3, A1 $>$ U3 > U1 \\
\hline
\end{tabular}

Note-U1 = unassociated nouns, $1-\sec$ presentation; $U 3=u n$ associated nouns, 3-sec presentation; $A I=$ associated nouns, 1 -sec presentation; and $A 3=$ associated nouns, $3-\sec$ presentation. 
and 14-year-olds. For 17-year-olds, associative structure alone resulted in highest recall accuracy with dichotic presentation. When words were presented monaurally, however, the associative structure variable was clearly the dominant organizational cue; presentation rate was secondary. Thus, all age groups were able to organize material, utilizing associative structure, more effectively when information was presented monaurally. Under dichotic presentation, only $17-y e a r-o l d s$ indicated the dominant effects of associative structure. Fourteen-year-olds, although indicating the combination of variable effect under dichotic presentation characteristic of younger Ss (associated, 3-sec > associated, 1-sec, unassociated, 1- and 3-sec presentation rates), also performed significantly better under associative conditions (associated, 1-sec $>$ unassociated, 1- and 3-sec presentation rates).

The strategy analyses of dichotic and monaural presentation of word lists indicated that the Ss were able to employ list structure for organization of information (Friedrich, in press, a, b). Under associated, 1- and 3-sec presentation and unassociated, 3 -sec presentation rate conditions, the back-to-front strategies were employed by all Ss; adolescents augmented back-to-front strategies with front-to-back (rehearsal) strategies. Under the dichotic unassociated, 1-sec presentation rate condition, the ear backward strategies were employed by all Ss when minimal structure was available. All age groups employed optimal strategies less frequently and less efficient strategies more frequently under the unassociated, 1 -sec presentation rate condition. Friedrich (in press, a,b) found that, with both presentation procedures, 7- and 10-year-olds did not employ optimal strategies as frequently as older Ss. Further, both age groups were less accurate in recall, regardless of strategy use, than adolescents. These findings are reflected in the depressed serial position curves for children, relative to adolescents.

An important conclusion to be drawn from the dichotic-monaural presentation comparison is that all age groups, to varying degrees, indicate appropriate strategy use under changing experimental conditions, regardless of presentation procedure. Thus, although age differences were found in number of words recalled and frequency of strategy employment, all age groups did consistently incorporate list cues across monaural and dichotic presentation procedures for information organization.

It is interesting to note the similarity of the dichotic and monaural curves for each age group under varying conditions in relation to storage capacity and control process findings of Friedrich (in press, $a, b$ ). That is, 7 . and 10-year-olds indicated a more limited short-term memory capacity (dichotic presentation) and undeveloped control processes (dichotic and monaural presentations) than older Ss. As is illustrated in Figs. 1.4, both monaural and dichotic curves for children, relative to adolescents, are depressed at the beginning and middle serial positions. This curve depression has been interpreted as a result of undeveloped control processes for transferring information from short-term to long-term memory. These crossexperimental findings suggest the following between-age conclusions. Middle childhood Ss have a more limited short-term memory capacity than older Ss for encoded information presented dichotically but not monaurally. Under dichotic presentation, the youngest $\mathrm{Ss}$ recalled significantly less information than older Ss, even when using the optimal orders of report characteristic of the latter group. In addition, 7-year-olds employed optimal strategies less frequently and less efficient strategies more frequently than older children and adolescents, regardless of presentation procedure. Under monaural presentation conditions, the primacy effect is not observable for 7-year-olds. This finding suggests that middle childhood Ss, employing the back-to-middle strategy, have not developed adequate strategies or control processes for transferring information (encoded serially) from short-term to long-term memory. The 10-year-olds also exhibited a more limited short-term memory capacity than adolescents for dichotically presented words. Here again, late childhood Ss recalled significantly less information than adolescents, even when using the optimal strategies characteristic of the latter group. When information is presented monaurally, 10-year-olds indicated undeveloped control processes (reflected in order of report). That is, under monaural conditions, optimal strategies were supplemented with moderate use of the less efficient random and back-to-middle strategies.

The between-age dichotic and monaural serial position curve comparisons support the delineation of memory structure and control process age-related change of Friedrich (in press, a, b). For dichotic presentation, middle and late childhood Ss were less accurate in recall than adolescents, regardless of strategy use. This finding suggests a capacity limit change through childhood (7-year-olds more restricted than 10-year-olds) and adolescence. The child's relative lower accuracy of recall, even with the optimal strategies used more frequently by adolescents, may be due, in part, to a restricted storage limit for relevant information. This conclusion is based on the notion that, if a child indicates strategy use typical of adolescents, any difference in word recall for a similar strategy that favors the latter group is the result of a more limited capacity of the former group. Under both dichotic and monaural presentations, the childhood Ss (7-year-olds to a greater extent than 10-year-olds) did not use the optimal strategies for the various structure cues as frequently as adolescents. Further, children used less efficient strategies more frequently than adolescents. These findings support the hypothesis of age-related changes in memory processes. Again, the depression of serial position curves for children for beginning and middle positions reflects the age-related quantitative change in memoric control processes. 
Finally, the striking similarity of dichotic and monaural serial position curves within age groups supports the proposal of similar strategy use within ages across presentation procedures and the use of similar orders of report in plotting serial position curves for dichotic and monaural free recall tasks. That is, the curve similarities within ages for both presentation procedures reflect the similarity of orders of report and amount of recall in dichotic and monaural free recall tasks.

\section{REFERENCES}

Atkinson, R. C., \& Wickens, T. D. Human memory and the concept of reinforcement. Technical Report 145 , Institute for Mathematical Studies in the Social Sciences, Stanford University, 1969.

Bryden, M. P. Binaural competition and division of attention as determinants of the laterality effect in dichotic learning. Canadian Journal of Psychology, 1969, 23, 101-105.

Friedrich, D. A primer for developmental psychology. Minneapolis: Burgess, 1972.

Friedrich, D. Developmental analysis of memory capacity and information encoding strategy. Developmental Psychology, in press, $a$.

Friedrich, D. Developmental analysis of memory strategy. Journal of Genetic Psychology, in press, b.

Glanzer, M. Distance between related words in free recall: Trace of the STS. Journal of Verbal Learning \& Verbal Behavior 1969, 8, 105-111.
Kintsch, W. Models for free recall and recognition. In D. A. Norman (Ed.), Models of human memory. New York: Acad em ic Press, 1970.

Palermo, D. S. Characteristics of word association responses obtained from children in Grades 1-4. Paper presented at the 1965 meeting of the Society for Research in Child Development, Minneapolis, Minnesota.

Palermo, D. S., \& Jenkins, J. J. Word association norms: Grade school through college. Minneapolis: University of Minnesota Press, 1964.

Shiffrin, R. M., \& Atkinson, R. C. Storage and retrieval processes in long-term memory. Psychological Review, 1969, 76. 179-193.

\section{NOTES}

1. A number of other restrictions were imposed on the selection of the second member of the pair that eliminated the following: (a) proper nouns, (b) plurals, (c) rhymes of the stimulus word, (d) words with more than three syllables, (e) words with a Thorndike-Lorge frequency less than four per million, (f) words given with any. sizable frequency $(\mathrm{N}>9$ ) as an association, within the norms, to any other first member, and (h) words identical to any member of the 64 pairs.

2. Response latencies were not recorded. The author is aware of the problem of the use of the word "immediate" in describing recall of end list words in the $B, B M$, and BMF strategies. Observing the Ss' performance, the recall of end list words in these orders of report was rapid, followed by a pause before recall of words from the middle or beginning of the list.

(Received for publication March 25, 1974; accepted April 12, 1974.) 